\title{
THE LACK OF FUNDAMENTAL ATOMIC AND MOLECULAR DATA: A CRISIS IN SPACE ASTRONOMY?
}

\author{
FREDERICK C. BRUHWEILER \\ Dept. of Physics \\ Catholic University of America \\ Washington, DC, U.S.A.
}

A crisis is now confronting space astronomy. Many current and future investigations at UV and Extreme-UV wavelengths with space observatories such as the International Ultraviolet Explorer (IUE), Hubble Space Telescope (HST), Extreme UV Explorer, and Lyman are limited by the current availability of good fundamental atomic data such as accurate energy levels, wavelengths, and oscillator strengths.

Until very recently, spectroscopic studies using the $I U E$ of hot $\mathrm{O}$-subdwarfs have revealed the vast majority of the photospheric features could not be identified. Our recent detection of species such as Fe V, VI, and VII in very hot white dwarfs was unexpected and has definite implications for observations to be obtained by the EUVE and Lyman. Coadded spectra of these degenerate objects show many other unidentified features. Accurate atomic data for these highly ionized species will be a prerequisite to doing any quantitative analysis of these species and understanding the flux distributions in the EUV, where the strongest lines of these highly-ionized species are located.

Recent data obtained by the Goddard High Resolution Spectrograph aboard the HST also pose a strong challenge to the spectroscopist. The high resolution and signal-to-noise capabilities of this spectrograph enables one to determine wavelengths to an accuracy of 2-3 $\mathrm{x} 10^{-3} \AA$ and abundance estimates from weak interstellar lines to better than 10 percent, and ofter better than most of the uncertainties in the oscillator strengths used. Moreover, with the GHRS we should now have the ability to probe the UV molecular absorption of, in addition to $\mathrm{CO}$, such species as $\mathrm{CH}_{2}, \mathrm{HCl}, \mathrm{CS}$ and $\mathrm{H}_{2} \mathrm{O}$, for which reliable fundamental data will be needed.

Due to the funding limitations and the overwhelming need for new, more accurate atomic data, a very close working relationship between the laboratory spectroscopist and the astrophysicist is essential. Indeed, just such a cooperative effort has allowed us to identify most of the strong photospheric features in the hottest stars in the $\lambda \lambda 1150-2000$ range. Partially spurred by the above problems, especially in spectra of hot stars, we have initiatied such a close working relationship with laboratory spectroscopists of Univ. of Lund in Sweden. In essence, we take advantage that both the astronomical and laboratory spectra show many unidentified lines. In this project, we use $I U E$ high resolution spectra of O-subdwarfs of different surface temperatures as "ionization filters" as an aid in identifying 
unidentified features in individual laboratory spectra, which often show several stages of ionization simultaneously. Unidentified, strong features in a particular O-subdwarf must arise from an ion within a specified range of ionization energy. The measured wavelength of these lines are then compared to the laboratory spectra in an attempt to establish coincidences in the laboratory data for ions within the required ionization range. This makes the laboratory identification process much easier and allows the energies of additional levels to be determined, plus it concentrates the efforts on only the transitions that are important to astrophysics. With improved energy levels, reliable oscillator strengths are then calculated. Our goal is to greatly expand the database of reliable atomic data for hot stars.

Close cooperation between laboratory spectroscopists and astrophysicists will be extremely important in the future. Similar joint projects like that described above should conducted. Thus, with a little ingenuity, we can collectively make use of the limited resources that are available. 